\title{
A Deep Learning Approach for UAV Enabled NOMA Relaying System
}

\author{
Messiah Josephine $\mathrm{M}^{\mathrm{a}, 1}$, Ameelia Roseline $\mathrm{A}^{\mathrm{b}}$ \\ ${ }^{a} P G$ Scholar, Dept of ECE, Panimalar Engineering College, Chennai \\ bProfessor, Dept of ECE, Panimalar Engineering College, Chennai
}

\begin{abstract}
The Increasing Demand in Wireless Devices Leads to Low Data Rate and Low Efficiency to resolve this Problem 5g is evolved. NOMA Technique is proposed to Face Challenges and Difficulties Issues in $5 \mathrm{~g}$ Networks. Same frequency spectrum can used by more than one user is a major advantage in NOMA. The LMMSE Algorithm with NOMA is proposed In this Research. thus by LMMSE with OFDM is Compared. The Inter Channel Interference Using Equalizer Results In NOMA than OFDM In The Future Research Novel Deep Leaning using UAV enabled NOMA will be analyzed.
\end{abstract}

Keywords. NOMA, LMMSE, Zero forcing, 5G

\section{Introduction}

Wideband transmission frameworks for constant payload information correspondences have gotten almost no consideration up to this point. The possibility of all while accepting the numerous client signals and identifying them isn't new; its history returns to the 1970s. At that point, Verdu found the ideal multiuser beneficiary of CDMA frameworks during the 1980s, which depends on the most extreme probability collector recognizing various clients in equal. We take CDMA for instance to show MUD. As opposed to apportioning symmetrical RBs to various clients as in FDMA, TDMA and CDMA codes the signs of numerous clients with the guide of one of a kind, client explicit mark introduced as spreading codes. On the off chance that we utilize symmetrical chip, we can just help clients. In any case, when utilizing non-symmetrical arrangements in the soul of NOMA. Usually, the multiuser IC can be separated into twofold fundamental classifications, to be specific pre-interference cancelation (pre-IC) and post interference cancellation (post-IC)[6]. All the explicitly, pre-IC procedures are utilized at the receiver side. The post-IC procedures are utilized at the recipient site for dropping the impedance [7]. On the off chance that we complete exact force control to guarantee that every got sign is comparable, PIC beats SIC. Paradoxically, SIC works better, when they got forces are diverse on the grounds that the most grounded client's sign can be identified first. The distinguished piece is the re-modulate by successive request to clear most vulnerable sign [8]. It is important that in the complexity tradeoff, there is additionally an assortment of different tradeoffs among PIC and SIC. There are likewise some ongoing IC systems [9]. Concerning LTE heterogeneous systems (HetNets), a few organizations, for example, Qualcomm and Fujitsu have likewise grown amazing IC procedures [10].

\footnotetext{
${ }^{1}$ Messiah Josephine M, PG Scholar, Dept of ECE, Panimalar Engineering College, Chennai, India; E-mail: messiahjosephine@gmail.com
} 


\section{Related Works}

Y. Saito et.al [1] researches the framework level execution of non-orthogonal multiple access (NOMA) with a progressive impedance canceller (SIC) on the collector side. The objective is to explain the potential of improving NOMA in over orthogonal multiple access (OMA). ). In light of PC reenactments, we appear under different designs that the framework level execution accomplished by NOMA is better than that for OMA. Utilizing PC recreations, we demonstrated the general throughput, client throughput, and the level of NOMA are for the most part better than that for OMA. Right now, encouraging innovation, Non-Orthogonal Multiple Access (NOMA), is examined, which statement a portion of problems for 5G. NOMA can suit substantial more users of non-orthogonal resource allocation. L. Dai et.al [2] They proposed an idea of Software-Defined Multiple Access ( SoDeMA) can able to diverse services and applications with various necessities. It is usual that NOMA will assume a significant job in future $5 \mathrm{G}$ remote correspondences.In view of the standards of NOMA and Full-Duplex (FD) correspondence, H. Huang et.al [3] proposed a novel FD-bolstered supportive NOMA (FD-NOMA) plan to upgrade the most extraordinary attainable rate area. At the present time, the greatest attainable rate area reliant on NOMA has been considered. A FD-NOMA model with three rate region headway plans has been proposed. We presume that via cautiously picking the force designation factor and the weight coefficient, the greatest attainable rate district can be gotten past our desires. The new NOMA-SM(spatial multiplexing) has been proposed . Y. Chen et.al[4].Its BER performance explore the influence of the Rician $K$-factor, spatial correlation of antenna array, time-varying effect of the V2V channel, and the power allocation factor is discussed. In instantaneous, NOMA-SM has discovered to expand the link reliability and bandwidth efficiency of V2V communications Z. Wei et.al [5] proposed a suboptimal iterative resource allocation calculation dependent on the contrast of convex programming.

\section{Existing Exhaustive Search Method For Channel Estimation For OFDM}

OFDM channel has strategy for accessing shared medium systems. It limits a few users to have similar Frequency channel by separating the sign into multiple time slots. The users transmit in fast progression, each utilizing its way of scheduling vacancy. In the current framework, they consider vitality productive transmissions for OFDM arrange in which the auxiliary clients exist together with the essential clients [11]. The joint time scheduling and beam-forming improvement is non-convex and is regularly profoundly complex to settle. we show that the ideal time portion and the ideal beam-forming vectors can be discovered productively in polynomial-time through a proper decomposition. The major disadvantages of existing system Cochannel interference happens.

\section{Proposed NOMA (Non-Orthogonal Multiple Access in 5g)}

In this paper we have two phase of simulation, one is $5 \mathrm{G}$ application with NOMA techniques, another one is Deep learning approach enabled UAV, first we have simulated the results for 5G-NOMA with the proposed LMMSE, Zero forcing 
algorithms. Second phase can be implemented for future research. At present scenario, for the high date rate transmission we have chosen the application 5G-LTE. In order to overcome the challenges and difficulties of 5G-LTE NOMA is the promising one. Learning method is proposed that automatically investigates the channel state information (CSI) of the communication system and detects the original transmit sequences.

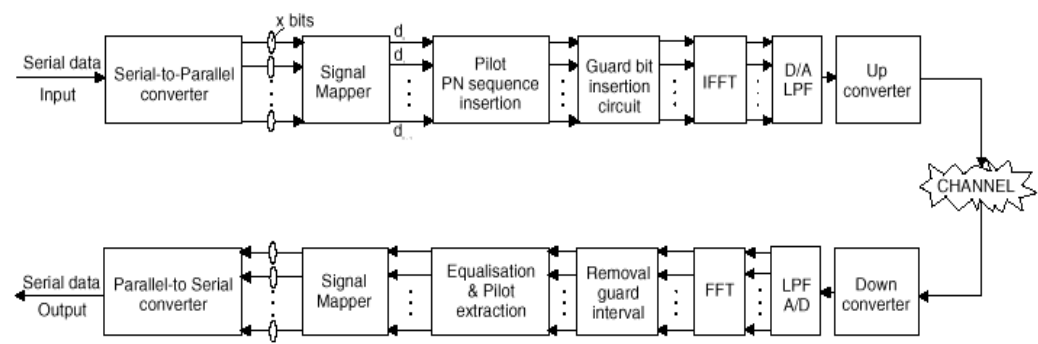

Figure 1. Block diagram for 5g-LTE

\subsection{Zero forcing algorithm}

Zeroforcing algorithm can be called as a null interference algorithm, used in communi cation systems that nullify the interference signal in the time domain or invert the chan nel frequency range in the frequency domain.

Step1: The overall response at the input of the detector must follow the Nyquist criterion for no ISI:

$$
\begin{aligned}
G_{r c}(f) & =|P(f)| \cdot|C(f)| \cdot|H(f)| \cdot|E(f)| \\
& =G_{r c}(f) \cdot|C(f)| \cdot|E(f)| \Rightarrow \\
E(f) & =\frac{1}{|C(f)|} \cdot e^{-j a(f)} \text { where } a(f) \text { is the phase of } C(f)
\end{aligned}
$$

Step2: noise variance at the output of the equalizer is

$$
\sigma_{e}^{2}=\frac{N_{0}}{2} \cdot \int_{-\infty}^{\infty} \frac{G_{r c}(f) \mid}{|C(f)|^{2}} d f
$$

Step3: For no ISI, let

$$
g(k T)= \begin{cases}1 & k=0 \\ 0 & k= \pm 1, \pm 2, \cdots, \pm N\end{cases}
$$

\subsection{Proposed NOMA-MMSE Estimator Algorithm}

A Shortened LMMSE Channel Estimation Algorithm for OFDM Systems. It is notable that the linear least mean square error calculation is a one of the best other linear receivers OFDM channel estimation, which gives better mean squared errors (MSE) execution yet requires a larger number of calculations than others. The proposed NOMA-MMSE estimator algorithm can be explained as follows, 


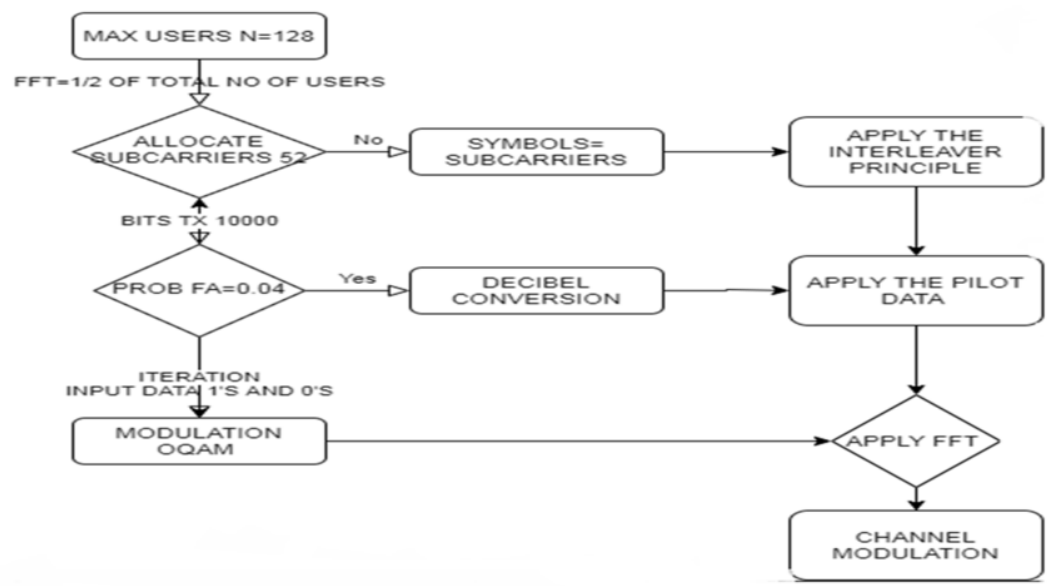

\section{Simulations And Discussion}

The outputs are simulated for the proposed NOMA-deep learning using MATLAB software.

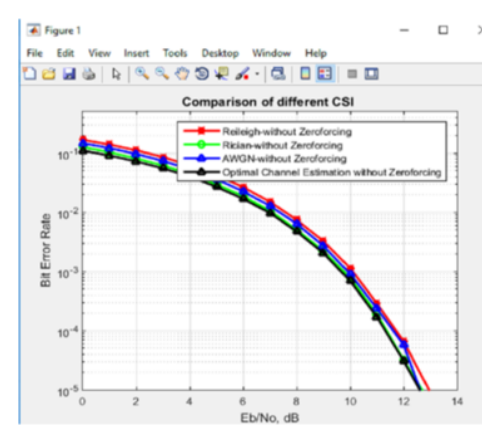

Figure 2. Comparison of different CSI

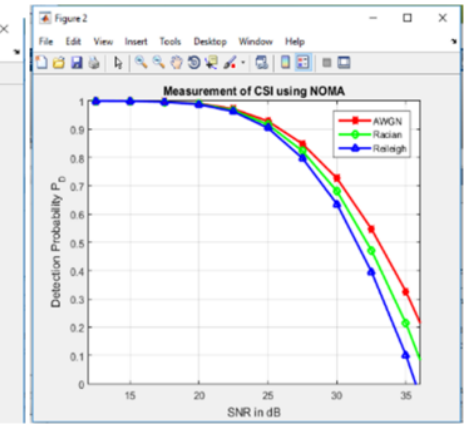

Figure 3.Measurement of CSI using NOMA

In Fig 2 shows the comparison of different channel state information with comparison of reileigh ,rician,AWGN, optimal channel estimation without zeroforcing the different channel compares with bit error rate and signal to noise ratio.Fig .3 shows that the measurement of channel state information using proposed NOMA with the comparison of three different channel AWGN,rician,reileigh with detection probability denotes $\mathrm{P}$ and signal to noise ratio in db. Fig.4 indicates that the it compares the bit erorr rate and signal to noise ratio for the proposed NOMA with ICI reduction using equalizer least minium mean square error ofdm with ICI reduction equalizer LMMSE NOMA. 


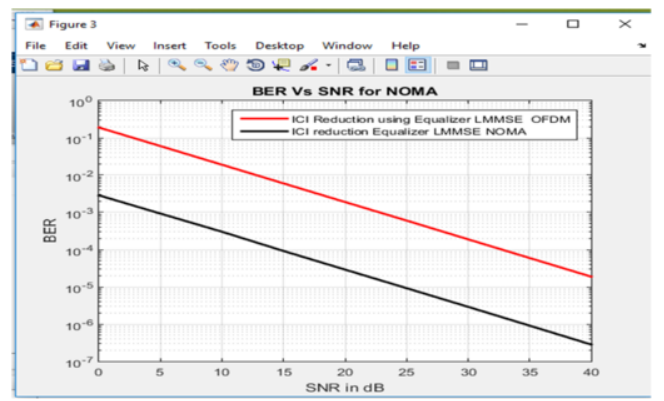

Figure 4. BER Vs SNR for NOMA

\section{Conclusion}

This paper proposed a $5 \mathrm{~g}$ with non-orthogonal multiple techniques and the simulation results are compared with channel state information with three different channels and compare it without zeroforcing. we can conclude that channel state information for NOMA is better than OFDM in [11]. Therefore NOMA is one the promising techniques for $5 \mathrm{G}$ challenges.

\section{References}

[1] Y. Saito, A. Benjebbour, Y. Kishiyama, and T. Nakamura, Systemlevel performance evaluation of downlink non-orthogonal multiple access (NOMA). in Proc. IEEE Annu. Symp. Pers. Indoor Mobile Radio Commun., London, U.K., Sep. 2013, pp. 611-615.

[2] L. Dai, B. Wang, Y. Yuan, S. Han, C. 1. I, and Z. Wang, Non-orthogonal multiple access for 5G: Solutions, challenges, opportunities, and future research trends. IEEE Commun. Mag., vol. 53, no. 9, pp. 74-81, Sep. 2015.

[3] H. Huang, J. Xiong, J. Yang, G. Gui, and H. Sari, "Rate region analysis in a full-duplex-aided cooperative non-orthogonal multiple-access system," IEEE Access, vol. 5, pp. 17869-17880, 2017.

[4] Y. Chen, L. Wang, Y. Ai, B. Jiao, and L. Hanzo, "Performance analysis of NOMA-SM in vehicle-tovehicle massive MIMO channels," IEEE J. Sel. Areas Commun., vol. 35, no. 12, pp. 2653-2666, 2017

[5] Z. Wei, D. W. K. Ng, J. Yuan, and H. M. Wang, Optimal resource allocation for power-efficient MCNOMA with imperfect channel state information. IEEE Trans. Commun., vol. 65, no. 9, pp. 3944 3961, Sep. 2017.

[6] Z. Ding, Z. Yang, P. Fan, and H. V. Poor, On the performance of nonorthogonal multiple access in 5G systems with randomly deployed users. IEEE Signal Process. Lett., vol. 21, no. 12, pp. 1501-1505, Dec. 2014.

[7] L. Yang, J. Chen, Q. Ni, J. Shi, and X. Xue, "NOMA-enabled cooperative unicast-multicast: Design and outage analysis," IEEE Trans. Wireless Commun., vol. 16, no. 12, pp. 7870-7889, Dec. 2017.

[8] J. A. Oviedo and H. R. Sadjadpour, A fair power allocation approach to NOMA in multiuser SISO systems. IEEE Trans. Veh. Technol., vol. 66, no. 9, pp. 7974-7985, Sep. 2017.

[9] B. Wang, L. Dai, Z. Wang, N. Ge, and S. Zhou, Spectrum and energy efficient beamspace MIMONOMA for millimeter-wave communications using lens antenna array.IEEE J. Sel. Areas Commun., vol. 35, no. 10, pp. 2370-2382, Oct. 2017.

[10]X. Yue, Y. Liu, S. Kang, A. Nallanathan, and Z. Ding, Outage performance of full/half-duplex user relaying in NOMA systems. in Proc. IEEE Int. Conf. Commun., Paris, May 2017, pp. 1-6.

[11] M.Messiah Josephine, A.Ameelia Roseline .Radio Resource Management For Femto Cell Wireless Network Based On MIMO-OFDM Communication.International Journal of Innovative Technology and Exploring (IJITEE) ISSN:2278-3075,volume-9 Issue-1S,November 2019. 